\title{
Comportamiento de los estreptococos beta-hemolíticos en escolares
}

\author{
Miranda García M. ${ }^{\mathrm{a}} \mathrm{C} .{ }^{1}$
}

Sanid. mil. 2012; 68 (1): 17-21; ISSN: 1887-8571

\begin{abstract}
RESUMEN
Introducción: Los estreptococos son responsables de diversos procesos infecciosos en nuestra comunidad, algunos de ellos de importante repercusión como fiebre reumática, endocarditis, glomerulonefritis, alopecia o escarlatina, que pueden suponer incluso epidemias en comunidades que conviven cercanas como los escolares. Objetivos: El objetivo de este estudio ha sido estudiar la frecuencia y variabilidad de los distintos grupos de estreptococos beta-hemolíticos en escolares portadores (12-18 años) de diferentes colegios de la población de San Fernando, así como la sensibilidad antibiótica de los microorganismos detectados. Material y Método: Se tomaron muestras a 160 escolares de ambos sexos que fueron sembradas e incubadas durante 48 horas. Se realizó coloración de Gram, prueba de catalasa, y sensibilidad a bacitracina. Los serogrupos se identificaron mediante pruebas de aglutinación, utilizando el kit Oxoid. Para la sensibilidad se utilizaron discos Becton-Dickinson con diversos antibióticos. Resultados: Se obtuvieron 34 muestras positivas en las que se aislaron 6 cepas de estreptococo beta-hemolítico grupo A, (3,75\%), 2 cepas del grupo B, 5 del grupo C, 10 del grupo F, 6 del grupo G y 5 no agrupables (a los que dimos importancia por la abundancia de colonias crecidas que presentaban en placa). No siendo especialmente significativo, encontramos que hay una frecuencia mayor de estreptococos de los grupos A y $\mathrm{G}$ en varones y de los grupos $\mathrm{C}$ y $\mathrm{F}$ en mujeres. Conclusiones: La frecuencia de los estreptococos beta-hemolíticos de los grupos B, C, F, G y los no agrupables aislados, presentan un porcentaje mayor con respecto a los estudios realizados en otras ciudades. Respecto a las cepas de $S$. pyogenes estudiadas, encontramos una tasa de sensibilidad y resistencia similar a la de las ciudades descritas, pero menor que la del estudio realizado en Valencia en el 2002.
\end{abstract}

PALABRAS CLAVE: Estreptococos beta-hemolíticos, Escolares portadores, Sensibilidad.

\section{Beta-hemolytic streptococci in school children}

\section{SUMMARY}

Introduction: Streptococci are responsible for different infectious diseases in the community; some of them with serious consequences as rheumatic fever, endocarditis, glomerulonefritis, alopecia or scarlet fever, and have the potential to cause outbreaks in closed communities like school children. Objectives: the objective of this study was to determine the frequency and variability of the different groups of beta-hemolytic streptococci in carrier school children (12-18 years old) in different schools in the town of San Fernando, as well as their antimicrobial susceptibilities. Material and Methods: samples taken from 160 school children of both sexes were cultured and incubated for 48 hours. Gram stains, catalase tests and bacitracin sensitivity tests were carried out. Serogroups were identified by agglutination tests using the Oxoid kit. Antimicrobial susceptibility was determined with Becton-Dickinson discs with different antibiotics. Results: from 34 positive samples we isolated 6 group A beta hemolytic streptococci strains (3,75\%), 2 group B strains, 5 group C, 10 group F, 6 group G and 5 non-groupables (considered important due to the abundance of colonies on the plate). Although not significant we found a higher frequency of groups A and G streptococci in males and groups $\mathrm{C}$ and $\mathrm{F}$ in females. Conclusions: the frequency of the isolated groups B, $\mathrm{C}$, F, G and non-groupable beta hemolytic streptococci represents a higher percentage in comparison with studies carried out in other cities. As for the strains of S. Pyogenes we found antimicrobial susceptibility and resistance rates similar to those of other cities, but lower than that of the Valencia study in 2002.

KEY WORDS: Beta hemolytic streptococci, Carrier school children, Susceptibility.

\section{INTRODUCCIÓN}

La faringitis y faringoamigdalitis son algunas de las infecciones que con más frecuencia aparecen en la población pediátrica y adulta. Los virus son responsables del $80-90 \%$ de las faringoamigdalitis

${ }^{1}$ Técnico Especialista de Laboratorio. Hospital General de la Defensa San Carlos. Laboratorio de Microbiología. San Fernando. Cádiz. España.

Dirección para correspondencia: María Caridad Miranda García. Hospital General de la Defensa San Carlos. Laboratorio de Microbiología. C/ Capitán Conforto s/n. San Fernando 11.100 - Cádiz.

Recibido: 26 de noviembre de 2010

Aceptado: 1 de agosto de 2011 agudas, predisponiendo a sobreinfección bacteriana. Unas de las posibles infecciones de vías respiratorias altas con la misma etiología son otitis y sinusitis ${ }^{1}$. El principal causante de la faringoamigdalitis bacteriana aguda es el Streptococcus pyogenes (S. pyogenes) grupo A que puede ocasionar en la población escolar una infección aguda sin complicación, una epidemia o desencadenar asociada a la infección: fiebre reumática, endocarditis, glomerulonefritis, alopecia o escarlatina $^{2}$. Las faringoamigdalitis por estreptococos ocurren más a menudo en el calendario escolar, cuando grupos de personas se encuentran en espacios cerrados como son las aulas ${ }^{3}$. Se puede definir como infección estreptocócica a la presencia de la bacteria en el tracto respiratorio superior con clínica de faringoamigdalitis. El $S$. pyogenes produce una elevación de anticuerpos (Ac): ASLO. Estos 
Ac seguirán estando elevados durante un tiempo tras la remisión de la infección ${ }^{2}$. La identificación del género Streptococcus fundamentada por el tipo de hemólisis, antígeno polisacárido y reacciones bioquímicas son las que clasifican a los estreptococos. El S. pyogenes elabora la Streptolisina O, inhibiendo la movilidad leucocitaria y la fagocitosis y tiene efectos cardiodepresores. Las exotoxinas pirogénicas son responsables del exantema de la escarlatina ${ }^{3}$. También son causa de infecciones faríngeas los estreptococos del grupo C, F, y $\mathrm{G}$ respectivamente, e igualmente se pueden aislar estreptococos del grupo B pero con menos frecuencia. Los del grupo C presentan síntomas clínicos similares a los del grupo $\mathrm{A}$, in vivo producen amigdalitis pultácea además de glomerulonefritis asociada a infección faríngea e incluso a un aumento del título ASLO; in vitro producen en ocasiones colonias beta-hemolíticas de igual aspecto que las del grupo A. Los del grupo F y G pueden llegar a producir infecciones dentales y orales ${ }^{4}$. Hay ocasiones en las que, no presentando sintomatología se aísla la bacteria, encontrándonos entonces en presencia de portadores. El principal modo de demostrar la etiología estreptocócica es el cultivo faríngeo. El estado de portador asintomático de estreptococos beta-hemolíticos es frecuente en niños de edad escolar (5-15 años). El resultado de la interacción entre la bacteria y el huésped colonizado determina la permanencia de estado de portador, evolución hacia la enfermedad o hacia su erradicación.

Dependiendo de la zona geográfica y de la época del año, entre un $10-40 \%$ de escolares asintomáticos son portadores de $S$. pyogenes y éstos excepcionalmente pueden transmitirlo. Aunque es importante saber que aproximadamente un $5,66 \%$ de los portadores tienen más riesgo de episodios de faringitis aguda que los que no lo son ${ }^{4-5}$.

El tratamiento erradicador estaría indicado cuando: 1) El portador tenga antecedentes de fiebre reumática. 2) Exista un aumento de infecciones en la comunidad y 3) En personal que trabaje con enfermos crónicos ${ }^{5,6}$.

Debido al uso de antibióticos, dentro de los grupos de estreptococos beta-hemolíticos puede encontrarse un cambio en la sensibilidad de los mismos. Durante muchos años la penicilina ha sido considerada como el antibiótico de elección para el tratamiento de faringoamigdalitis causada por el $S$. pyogenes. Debido a las alergias a la penicilina y al aumento de fracasos terapéuticos los macrólidos junto a la clindamicina son la alternativa al tratamiento antibiótico con penicilinas; sin embargo la resistencia a la eritromicina y otros macrólidos (claritromicina, azitromicina), han aumentado en los últimos años con porcentajes según la zona geográfica entre un 20$30 \%{ }^{7}$. El principal mecanismo de resistencia es la alteración del sitio de acción mediante la enzima metilasa, que le confiere resistencia tanto a macrólidos como a lincosamidas (clindamicina, lincosamina) y streptograminas (fenotipo conocido como MLS). Recientemente se ha descrito otro mecanismo mediado por una bomba de eflujo activo que sólo afecta a macrólidos pero no a clindamicina $(\text { fenotipo } \mathrm{M})^{8}$.

\section{MATERIAL Y MÉTODOS}

Se tomaron muestras de exudado faríngeo a 160 escolares que no hubieran tenido faringoamigdalitis en 15 días anteriores y que no hubieran recibido tratamiento antibiótico en los siete días previos. Las muestras se tomaron con una torunda de algodón estéril previo

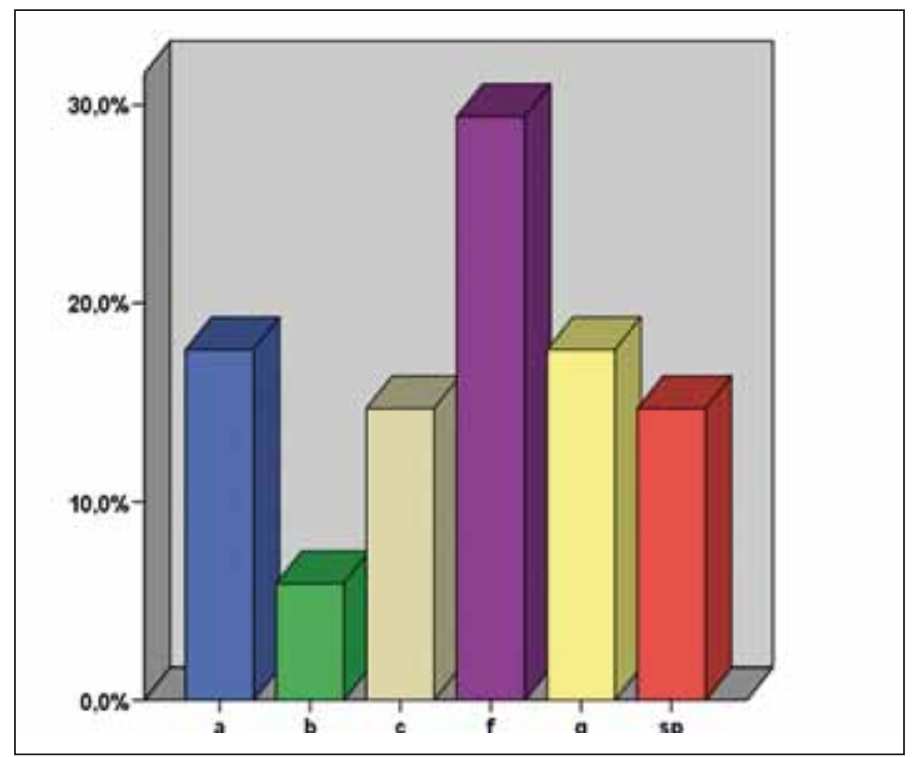

Figura 1. Porcentaje de crecimiento de grupos de estreptococos.

consentimiento por escrito de los escolares y de sus padres o tutores. El proyecto fue corregido y aprobado por el Comité Ético de Investigación Clínica de la Inspección General de Sanidad.

Las muestras fueron procesadas el mismo día de la toma. Fueron sembradas en agar sangre de carnero ANC (ácido nalidixico, colistina), incubándolas 48 horas a $37^{\circ} \mathrm{C}$, en atmósfera de $5 \%$ de $\mathrm{CO}_{2}$. A las colonias beta-hemolíticas crecidas se les realizó la coloración de Gram, prueba de la catalasa, y sensibilidad a la bacitracina. Para la identificación definitiva de los serogrupos se utilizaron pruebas de aglutinación, basadas en la detección de antígenos polisacáridos de los grupos A, B, C, F, y G utilizando un kit comercial (Oxoid). Para la sensibilidad se utilizaron discos de penicilina, eritromicina, ampicilina, amoxicilina-clavulánico y clindamicina (Becton-Dickinson).

\section{RESULTADOS}

De las 160 muestras recogidas 49,38 \% fueron mujeres y 50,62 \% varones. En 34 muestras $(21,25 \%)$ hubo crecimiento de estreptococos beta-hemolíticos, de las cuales el 47,06\% eran muestras de varones y el 52,94\% de mujeres. El resultado obtenido de las muestras estudiadas fue: 6 cepas aisladas de estreptococos grupo A $(17,64 \%), 2$ de grupo B $(5,88 \%), 5$ de grupo C (14,71\%), 10 de grupo $\mathrm{F}(29,41 \%), 6$ de grupo $\mathrm{G}(17,64 \%)$, y 5 no agrupables (sp) $(14,71 \%)$ (Figura 1$)$.

Con respecto a las edades y sexo de los aislamientos positivos, se puede observar el porcentaje en la tabla 1 . Se puede apreciar también que en las edades comprendidas entre 12-13, 15-16 y 17-18 años es mayor la frecuencia, 29,42\% (IC95\%:19,72-39,12) 26,47\%

Tabla 1. Aislamiento de estreptococos beta- hemoliticos por edades y sexo.

\begin{tabular}{|lccccccccc|}
\hline $\begin{array}{l}\text { Edad } \\
\text { (años) }\end{array}$ & $\mathbf{1 2 - 1 3}$ & $\mathbf{1 3 - 1 4}$ & $\mathbf{1 4 - 1 5}$ & $\mathbf{1 5 - 1 6}$ & $\mathbf{1 6 - 1 7}$ & $\mathbf{1 7 - 1 8}$ & Total & \% \\
\hline Varón & 6 & 2 & 0 & 6 & 0 & 2 & 16 & 47,06 \\
Mujer & 4 & 1 & 4 & 3 & 1 & 5 & 18 & 52,94 \\
Total & 10 & 3 & 4 & 9 & 1 & 7 & 34 & 100 \\
\hline
\end{tabular}




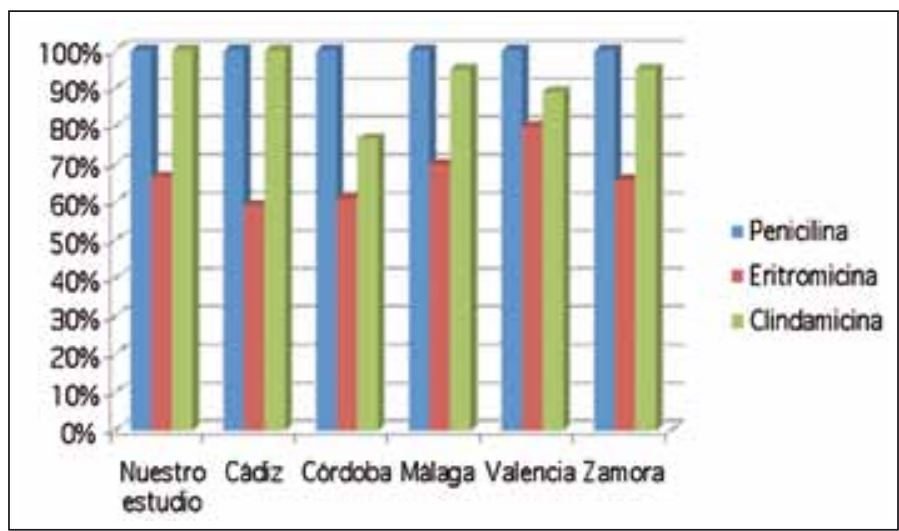

Figura 2. Sensibilidad del S. pyogenes en los diferentes estudios.

(IC95\%: 17,75-35,19) y 20,58\% (IC95\%:13,84-27,32) respectivamente.

La tabla 2 presenta los diferentes grupos de estreptococos betahemolíticos y su porcentaje a razón del sexo. Encontramos que hay una frecuencia mayor de los grupos A y $\mathrm{G}$ en varones, y de los grupos $\mathrm{C}$ y $\mathrm{F}$ en mujeres.

Encontramos una frecuencia mayor en el crecimiento de cepas de estreptococos grupo F: 29,41\% (IC95\%: 19,71-39,12).

En la tabla 3, se muestra el crecimiento de las diferentes cepas de estreptococos beta-hemolíticos en los cultivos faríngeos de los alumnos de distintas edades, y su porcentaje.

En cuanto a las sensibilidades la del estreptococo pyogenes a la penicilina no ha variado y según nuestro resultado sigue siendo ésta el tratamiento de elección en las faringoamigdalitis estreptocócicas con una sensibilidad del 100\%. Los estreptococos de grupo G y no agrupables tuvieron una sensibilidad de un 50\% y $20 \%$ respectivamente; los de los grupos B, C y F fueron resistentes; a la ampicilina todos fueron sensibles $(94,12 \%)$ excepto dos cepas del grupo $\mathrm{C}$ $(5,88 \%)$; para amoxicilina clavulánico tuvieron todos una sensibilidad del 100\%; para la eritromicina encontramos una sensibilidad de: $66,67 \%$ para el grupo A, $100 \%$ para el grupo B, $40 \%$ para el grupo C, $90 \%$ para el grupo F, $83,33 \%$ para el grupo $\mathrm{G}$ y $80 \%$ para los no agrupables; la resistencia de los distintos grupos fue de: $33,33 \%$

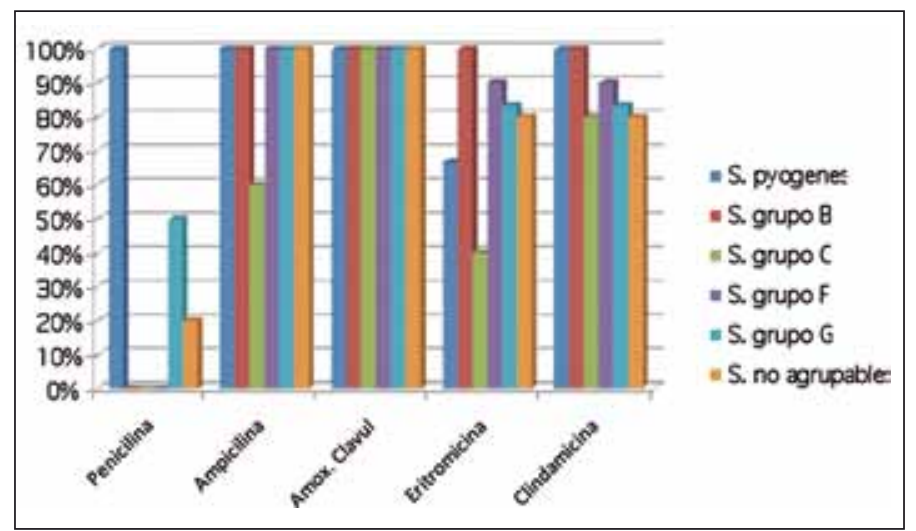

Figura 3. Sensibilidades de los diferentes grupos de estreptococos beta-hemoliticos.

para el grupo A, 60\% para el grupo C, 10\% para el grupo F, 16,67\% para el grupo G, y $20 \%$ para los no agrupables.

En la Tabla 4 apreciamos las sensibilidades y resistencias de los estreptococos beta-hemolíticos aislados y los porcentajes. (Ver también figura 3).

No se han podido establecer diferencias significativas entre los distintos grupos de estreptococos y la sensibilidad/ resistencia a los diferentes antibióticos (Figura 3).

\section{DISCUSIÓN}

Es un hecho conocido que el S.pyogenes es el patógeno más importante en las infecciones respiratorias de vías altas en niños, variando la tasa de estos estreptococos de una región a otra del mismo país. En este estudio hemos podido comprobar cómo el crecimiento total de cepas de estreptococos beta-hemolíticos en portadores sanos es de un $21,25 \%$, y de $S$. pyogenes la frecuencia es de un $17,64 \%$. En Málaga en un estudio sobre crecimiento de $S$. pyogenes en portadores sanos en el año 2006, la frecuencia encontrada fue entre un $15 \%-20 \%{ }^{12}$, y en Bilbao también en un estudio sobre escolares portadores la frecuencia fue de un $11,25 \%^{13}$. El porcentaje de estreptococos grupo B (5,88\%), grupo C (14,71\%), grupo F $(29,41 \%)$,

Tabla 2. Diferentes grupos de estreptococos beta-hemoliticos, sexo y porcentaje de los mismos.

\begin{tabular}{|lcccccc|}
\hline Grupo & A & B & C & F & G & No agrupable \\
\hline Varón & $4(11,76 \%)$ & $1(2,94 \%)$ & $1(2,94 \%)$ & $3(8,82 \%)$ & $4(11,76 \%)$ & $3(8,82 \%)$ \\
Mujer & $2(5,88 \%)$ & $1(2,94 \%)$ & $4(11,76 \%)$ & $7(20,5 \%)$ & $2(5,88 \%)$ & $2(5,88 \%)$ \\
Total & $6(17,65 \%)$ & $2(5,89 \%)$ & $5(14,70 \%)$ & $10(29,41 \%)$ & $6(17,65 \%)$ & $5(14,70 \%)$ \\
\hline
\end{tabular}

Tabla 3. Edades de los alumnos, diferentes grupos de estreptococos y el porcentaje de los mismos.

\begin{tabular}{|c|c|c|c|c|c|c|c|}
\hline Edad (años) & $12-13$ & $13-14$ & $14-15$ & $15-16$ & $16-17$ & $17-18$ & Total \\
\hline Grupo A & 4 & 1 & 1 & 0 & 0 & 0 & 6 \\
\hline Grupo B & 1 & 0 & 0 & 0 & 0 & 1 & 2 \\
\hline Grupo C & 1 & 0 & 0 & 1 & 0 & 3 & 5 \\
\hline Grupo F & 2 & 2 & 1 & 3 & 0 & 2 & 10 \\
\hline Grupo G & 1 & 0 & 1 & 2 & 1 & 1 & 6 \\
\hline No agrupables & 1 & 0 & 1 & 3 & 0 & 0 & 5 \\
\hline Total & 10 & 3 & 4 & 9 & 1 & 7 & 34 \\
\hline$\%$ & 29,41 & 8,82 & 11,76 & 26,47 & 2,94 & 20,59 & 100 \\
\hline
\end{tabular}


Tabla 4. Sensibilidades y resistencias de los estreptococos beta-hemoliticos.

\begin{tabular}{|c|c|c|c|c|c|c|c|}
\hline Grupo & $\mathbf{A}$ & B & $\mathrm{C}$ & F & $\mathbf{G}$ & No agrupable & Total \\
\hline \multicolumn{8}{|c|}{ Penicilina } \\
\hline $\mathrm{S}$ & $6(100 \%)$ & $0(0 \%)$ & $0(0 \%)$ & $0(0 \%)$ & $3(50 \%)$ & $1(20 \%)$ & $10(29,42 \%)$ \\
\hline $\mathrm{R}$ & $0(0 \%)$ & $2(100 \%)$ & $5(100 \%)$ & $10(100 \%)$ & $3(50 \%)$ & $4(80 \%)$ & $24(70,58 \%)$ \\
\hline \multicolumn{8}{|c|}{ Ampicilina } \\
\hline $\mathrm{S}$ & $6(100 \%)$ & $2(100 \%)$ & $3(60 \%)$ & $10(100 \%)$ & $6(100 \%)$ & $5(100 \%)$ & $32(94,11 \%)$ \\
\hline $\mathrm{R}$ & $0(0 \%)$ & $0(0 \%)$ & $2(40 \%)$ & $0(0 \%)$ & $0(0 \%)$ & $0(0 \%)$ & $2(5,89 \%)$ \\
\hline \multicolumn{8}{|c|}{ Amoxi/Clav } \\
\hline $\mathrm{S}$ & $6(100 \%)$ & $2(100 \%)$ & $5(100 \%)$ & $10(100 \%)$ & $6(100 \%)$ & $5(100 \%)$ & $34(100 \%)$ \\
\hline $\mathrm{R}$ & $0(0 \%)$ & $0(0 \%)$ & $0(0 \%)$ & $0(0 \%)$ & $0(0 \%)$ & $0(0 \%)$ & $0(0 \%)$ \\
\hline \multicolumn{8}{|c|}{ Eritromicina } \\
\hline $\mathrm{S}$ & $4(66,67 \%)$ & $2(100 \%)$ & $2(40 \%)$ & $9(90 \%)$ & $5(83,33 \%)$ & $4(80 \%)$ & $26(76,47 \%)$ \\
\hline $\mathrm{R}$ & $2(33,33 \%)$ & $0(0 \%)$ & $3(60 \%)$ & $1(10 \%)$ & $1(16,67 \%)$ & $1(20 \%)$ & $8(23,53 \%)$ \\
\hline \multicolumn{8}{|c|}{ Clindamicina } \\
\hline $\mathrm{S}$ & $6(100 \%)$ & $2(100 \%)$ & $4(80 \%)$ & $9(90 \%)$ & $5(83,33 \%)$ & $4(80 \%)$ & $30(88,24 \%)$ \\
\hline $\mathrm{R}$ & $0(0 \%)$ & $0(0 \%)$ & $1(20 \%)$ & $1(10 \%)$ & $1(16,67 \%)$ & $1(20 \%)$ & $4(11,76 \%)$ \\
\hline
\end{tabular}

grupo G $(17,64 \%)$ y no agrupables $(14,71 \%)$ encontrado en nuestro estudio es mayor que el encontrado en estudios realizados en otras comunidades, en los que la frecuencia de estos grupos es escasa. En Canarias un estudio realizado en el año 2000 demuestra que la tasa de portadores escolares de estreptococos beta-hemolíticos de grupo C y G (11\%-13\%) tienen una prevalencia más alta que los de grupo A $(6 \%)^{14}$, y en Venezuela en el estudio realizado el año 2002 en escolares asintomáticos los grupos B, F, y G también tuvieron una prevalencia más alta $(37 \%, 26 \%$ y $26 \%$ ) que el grupo A (21\%). Los grupos $\mathrm{C}$ y los no agrupables fueron del $14 \%$ y $7 \%$ respectivamente 4 .

Con respecto a la sensibilidad, durante años ha sido y sigue siendo la penicilina el tratamiento de elección para las infecciones producidas por S. pyogenes. Estudios realizados en otras comunidades y también el nuestro lo confirman ${ }^{9-11}$. No así para los estreptococos de grupos B, C, F, G, y no agrupables que, según nuestro estudio, presentan un porcentaje de sensibilidad a la penicilina inferior $(29,42 \%)$. La sensibilidad a ampicilina fue del $94,12 \%$ ya que dos cepas de estreptococo grupo C fueron resistentes $(5,88 \%)$; todas fueron sensibles a amoxicilina-clavulánico. Debido al hecho de que muchos pacientes aportan información confusa acerca de su posible alergia a penicilina, se prescriben como primera opción antibióticos macrólidos para el tratamiento de las faringitis o faringoamigdalitis bacteriana, por lo que se está observando un aumento de resistencia a los mismos.

En nuestro estudio la sensibilidad a eritromicina de las cepas de S. pyogenes fue del 66,66\% y la resistencia fue del 33,33\% (fenotipo $\mathrm{M}$ de resistencia); mayor en sensibilidad y menor en resistencia que los estudios realizados en Cádiz en el año 2000 en los que la sensibilidad a la eritromicina fue de $59,1 \%$ y la resistencia de $40,9 \%{ }^{9}$. En Córdoba, Málaga y Zamora estudios 1 levados a cabo en esas ciudades citan una tasa de sensibilidad entre el $61 \%$ y el $70 \%$ y una resistencia entre el $30 \%$ y el $39 \%{ }^{10,12,13}$. Comprobamos con esto que las cepas de $S$. pyogenes estudiadas tienen una tasa similar de sensibilidad y resistencia a las de las ciudades anteriormente descritas, pero menor que en Valencia cuyo estudio multicéntrico del 2002 la sitúa en una sensibilidad de $80 \%$ y una resistencia del $20 \%{ }^{11}$. En el resto de los estreptococos se encontró una sensibilidad del 78,66\%, y una resistencia del $21,34 \%$ en total.

En la figura 2 podemos apreciar la sensibilidad del $S$. pyogenes a penicilina, eritromicina y clindamicina, en los estudios de otras ciudades y en el nuestro.
En la figura 3 en la que se representan las sensibilidades de los estreptococos beta-hemolíticos según las cepas, podemos apreciar como respecto a eritromicina hemos obtenido:

- Estreptococos del grupo B sensibles a eritromicina (100\%).

- Estreptococos del grupo C: $40 \%$ sensibles y $60 \%$ resistentes.

- Estreptococos del grupo F : 90\% sensibles y 10\%resistentes.

- Estreptococos del grupo G: $83,33 \%$ sensibles y un $16,67 \%$ resistentes.

- Estreptococos no agrupables: $80 \%$ sensibles y $20 \%$ resistentes.

La resistencia a eritromicina se debe al uso en exceso de este antimicrobiano, si baja su uso, bajará también su resistencia ${ }^{10}$. En cuanto a sensibilidad y resistencia a clindamicina, hemos encontrado que todas las cepas de $S$. pyogenes son sensibles a la misma $(100 \%)$, un resultado igual al encontrado en $\mathrm{Cádiz}^{11}$, pero distinto al resto de las provincias mencionadas que sí encontraron resistencia a clindamicina (Figura 2). Para el resto de los estreptococos; los de grupo B fueron sensibles al 100\% y los de los grupos C, F, G, y los no agrupables al $80 \%, 90 \%, 83,33 \%$ y $80 \%$ respectivamente, y presentaron resistencias en el $20 \%, 10 \%, 16,67 \%$ y $20 \%$ respectivamente (en éstos tres últimos fenotipo MLS). Con respecto a la prevalencia y sensibilidad de estos grupos de estreptococos, observamos que también han experimentado un aumento en los estudios realizados en Canarias ${ }^{14}$ y Venezuela con una frecuencia de estreptococos de grupo B mayor que la nuestra (28,48\%), C y G similar (10,69\% y $19,85 \%)$ pero menor para el grupo $\mathrm{F}$ y el grupo de no agrupables (19,85\% y 5,34\% respectivamente). En relación a las sensibilidades, Venezuela tiene una sensibilidad mayor que la nuestra ${ }^{4}$. En general nuestros resultados concuerdan con los descritos en los estudios anteriores, aunque la prevalencia del grupo $\mathrm{F}$ sea superior y más afín con zonas de clima similar.

A la vista de los resultados obtenidos y considerando el número de portadores sanos, suponemos que una de las causas de la frecuencia con la que nos encontramos estreptococos beta-hemolíticos en la orofaringe de los escolares sea que esta población se encuentra en espacios cerrados como son las aulas. Además hemos visto que no sólo el $S$. pyogenes puede encontrarse en un portador asintomático, sino también pueden estar involucrados otros grupos de estreptococos betahemolíticos como los que hemos estudiado. Debido al elevado porcentaje de aislamientos de estreptococos betahemolíticos diferen- 
tes al de grupo A no debería considerarse al $S$. pyogenes como único estreptococo patógeno de la faringe, por lo que sería de mucha importancia identificar e informar todos los estreptococos beta-hemolíticos para un diagnóstico y tratamiento correctos y así evitar las lesiones no supurativas que las infecciones por éstos pudieran desencadenar.

Teniendo en cuenta todo esto, y sobre todo al S. pyogenes y sus posibles consecuencias tales como la fiebre reumática, endocarditis, glomerulonefritis, etc., se debería considerar el hacer más a menudo estudio de exudados faríngeos, cuyo resultado sería de ayuda a la hora de un diagnóstico que confirmara la etiología del agente causante y proporcionase la aplicación de un tratamiento correcto. En atención primaria no se suele realizar estudios microbiológicos, y se prescriben aproximadamente un $80 \%$ de antibióticos de manera empírica a los pacientes en un diagnóstico de presunción.

El tratamiento de elección sería penicilina o betalactámicos de espectro reducido, la amoxicilina constituye una alternativa razonable, en España es muy utilizada, bien tolerada por los pacientes y de mayor espectro que la penicilina y preferible por estas razones a amoxicilina-clavulánico y cefalosporinas aunque sea de mayor coste que la penicilina. Se reservan los macrólidos y lincosamidas para los casos de fracaso terapéutico, mal cumplimiento del tratamiento (ya que la posología y duración del tratamiento con penicilinas dificulta su cumplimiento y una mayoría de pacientes una vez que mejoran suspenden la medicación) y alergia demostrada a penicilina ${ }^{13}$.

Aunque en algunos estudios recomiendan tratamiento para erradicar estos microorganismos de la orofaringe de portadores ${ }^{4}$; otros no identifican con seguridad qué niños necesitan tratamiento y cuáles no lo necesitan ${ }^{13}$, un tercer estudio lo recomienda sólo en circunstancias especiales: historia familiar de fiebre reumática o contagio familiar de faringitis estreptocócica, puesto que el estado de portador puede permanecer durante meses, sin riesgo de desarrollar complicaciones, ni transmitir la infección ${ }^{15}$.

Considerando el estado de portador que en algún momento pueda presentar una faringoamigdalitis bacteriana, se ha de tener en cuenta todo lo expuesto anteriormente con respecto a un tratamiento antibiótico inadecuado, ya que la respuesta de los microorganismos a los antibióticos pudiera conllevar un aumento del nivel de resistencia a los mismos.

\section{CONCLUSIONES}

Los resultados obtenidos de nuestro estudio permiten concluir que, la frecuencia de los estreptococos beta-hemolíticos de los grupos B, C, F, G, y los no agrupables aislados en los escolares portadores, presentan un porcentaje mayor con respecto a los estudios realizados en otras ciudades. Con respecto a las cepas estudiadas de $S$. pyogenes encontramos que tienen una tasa de sensibilidad y resistencia similar a las de los estudios de las ciudades anteriormente descritas pero menor que la del estudio descrito en Valencia en el 2002.

\section{AGRADECIMIENTOS}

Al Tcol. D. Pelagio Fernández Sánchez jefe del Laboratorio de Microbiología por el apoyo y ayuda que me ha dado a la hora de hacer este estudio. A la Cte microbióloga $\mathrm{D}^{\mathrm{a}}$ Carmen González Gómez también por

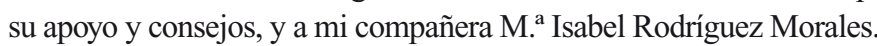

Al Comité Ético de Investigación Clínica de la Inspección General de Sanidad por su inestimable ayuda.

El material necesario para el estudio aprobado por la Pagaduría / Habilitación del Hospital General de la Defensa San Carlos.

\section{BIBLIOGRAFÍA}

1. Cervera Paz FJ. Clínica Universitaria de Navarra. Área de Salud. Faringitis crónica y aguda, 2009, www.cun.es /áreadesalud/.../faringitis- crónica- y aguda/

2. Guevara JM, Aguirre J, Valencia E, Willians F, Cuéllar E, Barboza M, Agusto W. Prevalencia de Ebh en pacientes con faringoamigdalitis aguda en un hospital de la ciudad de Chachapoyas. Amazonas. Anales de la Facultad de Medicina, Universidad Nacional Mayor de San Marcos. Lima. Perú. 2008; 69 (2): 88-90.

3. Diez O, Batista N, Bordes A, Lecuona M, Lara M. Diagnóstico microbiológico de las infecciones del tracto respiratorio superior. Hospital Universitario N $\mathrm{N}^{\mathrm{a}}$ Sra de la Candelaria. Sta.Cruz de Tenerife. Hospital de Gran Canaria Dr. Negrín. Las Palmas de Gran Canaria. Hospital Universitario de Canarias. La Laguna, Tenerife. Enfermedades Infecciosas Microbiología Clínica. 2007; 25: 387-93www.seimc. org./documentos/protocolos/.../cap23 asp.

4. Romero S, Ginestre M, Rincón G, Harris B, Martinez A. Estreptococo beta-hemolítico en la orofaringe de escolares asintomáticos de dos instituciones del Estado de Zulia. Cátedra de Microbiología, Cátedra de Bacteriología, Escuela de Bioanálisis Facultad de Medicina, la Universidad de Zulia, Maracaibo, Venezuela. Rev. Sociedad Venezolana de Microbiología. Caracas. Enero 2002; 22. (1): 6-11. ISSN 1315-2556.

5. Gianelli S y Bcqa, Posse G. Prevalencia de portación asintomática del Esteptococo Beta-hemolitico grupo A (Estreptococo pyogenes). Centro de Salud Provincial Puiggari. Secretaría de Investigación de la Facultad de Ciencias de la salud de la Universidad Adventista del Plata, Entre Ríos. Argentina. Cátedra de Microbiología Facultad de Medicina. Archivos argentinos de pediatría 2007; 105 (3): 221- 224. Buenos Aires.

6. Caballero J C, Vizcaya. Debate, portador sano de Estreptococo beta-hemolítico grupo A. 2006. grupos. emagister.com/.

7. Álvarez Alonso E, García Castro A, Sección de Microbiología Hospital General de Palencia Rio Carrión. Reunión de primavera de la Sociedad de Pediatría de Asturias, Cantabria, Castilla y León. Resistencia a antimicrobianos en Estreptococos pyogenes en el área de salud de Palencia. 2002; 42. (180):141-142.

8. Fariña N, Ocampos M.T., Laspina F, Balmaceda M A, Sanabria R, Samudio M. Estreptococo Beta-hemolítico grupo A, resistencia a los macrólidos. Departamento de Microbiología, Instituto de Investigaciones en Ciencia de la Salud, UniversidadNacional de Asunción. Laboratorio de San Roque. Asunción- Paraguay. www.iics.una.py/n/TEMA14.pdf.

9. Ochoa Sangrados C, Breznes Valdivieso M F, López-Urrutia Lorente L, Gutierrez Zufiaurrez M N, Varajas Sánchez M V, Bajo Delgado A F. Epidemiología de la infección estreptocócica faríngea en un área de salud. Bol Pediátrico. Servicio de Microbiología Hospital Virgen de la Concha. Zamora. 2006; 46: 32-38.

10. González-Lama Z, González J J, Lupiola P, Tejedor M T. Portadores de estreptococos beta-hemolíticos de los grupos A, B, C en escolares de Las Palmas. Servicio de Microbiología y Departamento de Ciencias Clínicas. ULPGC. Las Palmas. Enfermedades Infecciosas y Microbiología Clínica 2000; 18: 271-273.

11. Marín P, Márquez A, García-Martos P, García-Tapia A, y Mira J. Sensibilidad de Estreptococos pyogenes a los macrólidos y clindamicina en Cádiz. Servicio de Microbiología, Hospital Universitario Puerta del Mar, Cádiz.2002

12. Gordillo R M, Lacasa M S, Ibarra A, Rdguez F, Casal M. Sensibilidad al aislamiento faríngeo del estreptococo pyogenes en Córdoba. Servicio de Microbiología y parasitología, Hospital Reina Sofía de Córdoba. 2003.

13. García de Lomas J, López Cerezo L, Gimeno Cardona C y Gevipar. Sensibilidad de patógenos respiratorios en la comunidad en España: resultado del estudio Sauce. Instituto Valenciano. Departamento de Microbiología. Hospital Clínico Universitario de la Facultad de Medicina de Valencia. 2002.

14. Piédrola Maroto D, Montiel Quezol N, López Rdguez I, Monje Vega E, Casado Morente J C, Povedano Rdguez V, Fernández Ruiz E, Conde Jimenez M. Situación actual de las resistencias a antibióticos en las infecciones amigdalares. Unidad de ORL. Unidad de Microbiología. Hospital Costa del Sol. Marbella. Málaga. Acta Otorrinolaringología 2006; 57:171-175.

15. Cenjor C, García-RodriguezJ A, Ramos A, Cervera J, Tomás M, Asensi F, Cañada J L, Gobernado M, Isasiá T, López-Madroñero C, Martinez M, Pérez-Escanilla F, Picazo J, Sampelayo T. Documento de consenso sobre «tratamiento antimicrobiano de la faringoamigdalitis». Acta Otorrinolaringol. 2003; 54: 369-383. 